\title{
Lipiodol Trans-arterial Chemoembolization of Hepatocellular Carcinoma with Idarubicin: First Experience
}

\author{
Sylvain Favelier • Mathieu Boulin · Samia Hamza $\cdot$ Jean-Pierre Cercueil • \\ Violaine Cherblanc • Côme Lepage • Patrick Hillon • Bruno Chauffert • \\ Denis Krausé • Boris Guiu
}

Received: 8 October 2012/Accepted: 15 November 2012/Published online: 8 December 2012

(C) Springer Science+Business Media New York and the Cardiovascular and Interventional Radiological Society of Europe (CIRSE) 2012

\begin{abstract}
Background There is still no consensus about the best chemotherapeutic agent for transarterial chemoembolization (TACE). A recent in vitro study demonstrated that idarubicin, an anthracycline, was by far the most cytotoxic drug on human hepatocellular carcinoma (HCC) cell lines. Idarubicin is much more lipophilic than doxorubicin, leading to higher cell penetration through lipidic membranes and greater accumulation of the drug in the lipiodol. Furthermore, idarubicin has the ability to overcome multidrug resistance. Therefore, we designed this pilot human study to evaluate the safety and efficacy of lipiodol TACE using idarubicin.

Methods In 21 consecutive patients treated by lipiodol TACE with idarubicin $(10 \mathrm{mg})$ for HCC, safety data, tumor
\end{abstract}

S. Favelier · J.-P. Cercueil · V. Cherblanc · D. Krausé .

B. Guiu $(\square)$

Digestive, Thoracic and Oncologic Unit, Department of

Diagnostic and Interventional Radiology, University Hospital of

Dijon, 14, rue Paul Gaffarel, Dijon 21000, France

e-mail: boris.guiu@chu-dijon.fr

\section{Boulin $(\bowtie)$}

Department of Pharmacy, University Hospital of Dijon,

Dijon, France

e-mail: mathieu.boulin@chu-dijon.fr

\section{S. Hamza $\cdot$ C. Lepage $\cdot$ P. Hillon}

Department of Hepatology, University Hospital of Dijon,

Dijon, France

S. Hamza · J.-P. Cercueil · C. Lepage · P. Hillon · B. Guiu INSERM U866, University Hospital of Dijon, Dijon, France

B. Chauffert

Department of Oncology, University Hospital of Dijon,

Amiens, France response (Response Evaluation Criteria in Solid Tumors, mRECIST), time to treatment failure (TTTF), and overall survival were evaluated.

Results Postembolization syndrome was observed after $30.9 \%$ (17 of 55) of sessions. No patient died from a TACE-related complication. No hematological grade 3-5 adverse event was observed. At least one grade 3 or higher adverse event occurred in $19 \%$ (4 of 21) of patients. On imaging, no progression was encountered; four patients (24\%) exhibited stable disease, $12(57 \%)$ exhibited a partial response, and five (19\%) exhibited a complete response. Median TTTF was 16.7 months (Kaplan-Meier analysis). At 6 months, $94.7 \%$ (95\% confidence interval [CI] 68.1-99.2) of patients did not reach treatment failure, whereas treatment failure was not reached in $50.6 \%(95 \%$ CI 21.6-73.9) of patients at 1 year. Overall survival was $83.5 \%$ (95\% CI 57-94.4) at 1 year.

Conclusion Idarubicin seems safe and effective in lipiodol TACE of HCC. This warrants further study to determine the potential of this drug to replace doxorubicin for TACE.

Keywords Chemoembolization - Cirrhosis .

Liver cancer $\cdot$ Predictor $\cdot$ Survival

$\begin{array}{ll}\text { Abbreviations } \\ \text { TACE } & \text { Transcatheter arterial chemoembolisation } \\ \text { ECOG } & \text { Eastern Cooperative Group } \\ \text { HCC } & \text { Hepatocellular carcinoma } \\ \text { MDR } & \text { Multi-drug resistance } \\ \text { MRI } & \text { Magnetic resonance imaging } \\ \text { AST } & \text { Aspartate aminotransferase } \\ \text { ALT } & \text { Alanine aminotransferase } \\ \text { ALP } & \text { Alkaline phosphatises } \\ \text { GGT } & \text { Gamma glutamyltransferase }\end{array}$


NASH Non-alcoholic steatohepatitis

TTTF Time to treatment failure

OS Overall survival

$95 \%$ CI $95 \%$ Confidence interval

$\mathrm{AE} \quad$ Adverse event

\section{Introduction}

Hepatocellular carcinoma (HCC) is the fifth most common malignancy, and the third most common cause of cancerrelated death worldwide [1]. Because only $30 \%$ of patients can be treated for cure, palliative treatment options are applied in most cases [2]. For unresectable intermediatestage HCC, transarterial chemoembolization (TACE) plays a central role [3]. Although TACE has been demonstrated to prolong survival in two randomized, controlled trials and two meta-analyses [4], this procedure varies greatly across centers and interventional radiologists, especially regarding the anticancer drug chosen [5]. Only one randomized trial was designed to compare embolization alone versus TACE (vs. best supportive care) for HCC [6]. Unfortunately, this three-arm trial was prematurely stopped because TACE was demonstrated to be more efficient than best supportive care. Although there is currently no level 1 evidence on the benefit of chemotherapy in TACE, the recent introduction of drug-eluting beads has helped demonstrate a clear activity of chemotherapy in both animal $[7,8]$ and human $[9,10]$ studies, thanks to the comparison between loaded and unloaded beads.

One of the key theoretic advantages of TACE is tumor exposure to high concentrations of the chemotherapeutic drug [11]. Doxorubicin and cisplatin are the most widely used drugs for TACE [5], although no preclinical study or randomized, controlled trial has ever supported their use over that of other drugs. Because there is still no consensus about the best chemotherapeutic agent, we designed an in vitro study to screen for the best drug by comparing the cytotoxicity of multiple anticancer drugs on human HCC cell lines. This screening study demonstrated that idarubicin, an anthracycline commonly used to treat leukemias, was by far the most cytotoxic drug on three HCC cell lines [11].

HCC is considered to be one tumors most resistant to chemotherapy [12]. This is partly related to the multidrug resistance (MDR) mechanism, causing an increased ATPdependent efflux of drugs from within to outside the cells. MDR protein overexpression may account for the resistance to various drugs, including doxorubicin and cisplatin [13, 14]. Interestingly, besides its high cytotoxic effect, idarubicin has also the ability to overcome MDR [11].
Furthermore, idarubicin is much more lipophilic than doxorubicin [15], leading to higher penetration through the lipidic double layer of tumor cell membranes and thus has better efficacy. We can take advantage of this higher lipophilicity for TACE of HCC by combining idarubicin with ethiodized oil, which could result in a greater accumulation of the drug in the oily phase, permitting lipiodol to act as a slow-releasing vector.

To our knowledge, idarubicin has never been used intraarterially to treat liver tumors and especially HCC. Therefore, we designed this pilot human study to evaluate the safety and efficacy of lipiodol TACE using idarubicin.

\section{Patients and Methods}

\section{Patients}

The approval of the institutional review board was obtained to retrospectively evaluate the combination of idarubicin and lipiodol TACE of unresectable HCC. All patients provided informed consent for the TACE procedure.

Twenty-one consecutive patients treated by lipiodol TACE with idarubicin for HCC at our institution between June 2010 and March 2011 were enrolled onto this study. Indications for TACE were decided for all patients at our weekly multidisciplinary meeting of digestive oncology in the presence of interventional radiologists, gastroenterologists, hepatic surgeons, radiation therapists, and oncologists. Inclusion criteria were as follows: patients diagnosed by liver biopsy or noninvasive American Association for the Study of Liver Diseases criteria [16], Eastern Cooperative Oncology Group performance status of 0/1, preserved liver function (Child-Pugh class A or B7), platelet count of $>50 \times 10^{9} / \mathrm{L}$, and cardiac ejection fraction of $>50 \%$. Exclusion criteria were as follows: candidate for liver resection, biliary tract dilation, bilioenteric anastomosis, hepatofugal blood flow, thrombus within the main portal vein, extrahepatic metastases, serum creatinine level $\geq 150 \mu \mathrm{mol} / \mathrm{L}$ (renal failure), allergy to iodine-containing agents, pregnancy, and previous treatment by TACE.

Liver involvement was assessed on baseline magnetic resonance imaging (MRI) data obtained within 1 month before TACE. Baseline liver enzymes (aspartate aminotransferase [AST], alanine aminotransferase [ALT], alkaline phosphatases [ALP], $\gamma$-glutamyltransferase [GGT], total bilirubin, and prothrombin) were systematically obtained within 7 days before each TACE session.

\section{Lipiodol-Idarubicin Emulsion: In Vitro Evaluation}

The emulsion was prepared by mixing an equal volume of iodized oil (lipiodol; Guerbet, Aulnay-sous-Bois, France) 
and $1 \mathrm{mg} / \mathrm{mL}$ idarubicin (Zavedos; Pfizer, Paris, France) through a three-way tap from one $5-\mathrm{mL}$ syringe to another one (10 passages). The physical stability of the idarubicinethiodized oil emulsion was examined at $37{ }^{\circ} \mathrm{C}$. Thirty minutes after the preparation, the phase separation for idarubicin-ethiodized oil emulsion was limited $(5 \%$ aqueous solution and $95 \%$ persisting emulsion). The size distribution of the droplets of idarubicin-ethiodized oil emulsion was $20-100 \mu \mathrm{m}$, as measured by an inverted fluorescent microscope $\left(\lambda_{\text {excitation }}=485 \mathrm{~nm}\right)$ associated with AxoVision image analysis software for acquisition and image processing (Cell Observer; Carl Zeiss, Paris, France) (Fig. 1).

\section{TACE Procedure}

TACE was performed through a femoral access with a $5 \mathrm{~F}$ catheter. The patency of the portal branches was confirmed during the venous phase of an injection into the superior mesenteric artery. Then selective catheterization of the proper hepatic artery was performed. When accessory hepatic arteries were present, they were catheterized successively. Digital subtraction angiography and arterial computed tomographic imaging (when available) were used to plan the treatment. Depending on the degree of liver involvement, selective catheterization of the artery feeding the tumors was performed. As detailed above, a mixture of $10 \mathrm{mg}$ idarubicin and $10 \mathrm{~mL}$ iodized oil was injected in about $5 \mathrm{~min}$, followed by the injection of a particulate embolic agent of the radiologist's choice $(\mathrm{Cu}-$ raspon gelatin sponge; Curamedical B.V., Amsterdam, Netherlands) or unloaded beads (Embozene; Celonova Biosciences, Paris, France) until stasis. The initial dose of idarubicin was $10 \mathrm{mg}$, based on pharmacological data for anthracyclines. The cumulative cardiotoxicity of doxorubicin is observed at $550 \mathrm{mg} / \mathrm{m}^{2}$. The cumulative cardiotoxicity of idarubicin is observed at $93 \mathrm{mg} / \mathrm{m}^{2}$. Therefore, there is a 5.9:1 ratio between their cumulative doses. Because one of the most current (and also lowest) doses of doxorubicin used worldwide for TACE of HCC is $50 \mathrm{mg}$, we determined the initial dose of idarubicin as follows: $50 \mathrm{mg}($ doxorubicin $) / 5.9$ (ratio doxorubicin/idarubicin $)=$ $10 \mathrm{mg}$ idarubicin (approximately).

In cases of unilobar disease, the artery feeding the affected lobe was selectively catheterized with a microcatheter when needed $(2.7 \mathrm{~F}$ or $2.4 \mathrm{~F}$ Progreat; Terumo Europe, Leuven, Belgium). In case of bilobar tumor involvement, one lobe was treated during the first TACE session, while the other one was treated 6 weeks later. A cycle of TACE was defined as the one, two, or more sessions required to treat all liver nodules. Patients received systematic antiemetic medication with ondansetron $(8 \mathrm{mg})$ and on-demand pain medication.
Follow-up

Liver imaging was systematically performed by MRI (3 T Siemens Trio TIM, Erlangen, Germany). Baseline imaging was performed $<1$ month before each TACE session, and follow-up imaging was performed within 6 weeks after each session. Liver/biliary toxicity (dilated bile duct, portal vein branch narrowing, portal venous thrombosis, and biloma/liver infarct) was assessed on follow-up imaging, according to previously published data [17]. Repeat TACE procedures were performed on the basis of tumor response and patient health.

The primary end point was clinical and biologic tolerance according to the National Cancer Institute Common Terminology criteria (NCI-CTCAE) v4.0 [9]. For these patients, it was decided at our institution to prospectively and systematically collect grade 3-5 adverse events (AEs). Each patient was followed for 1 month after TACE. During this period, toxicities during the TACE procedure, the hospital stay, at the 1-month scheduled consultation or at emergency visits were recorded. During the TACE procedure, we monitored $\mathrm{O}_{2}$ saturation, electrocardiogram, arterial pressure, and pain. During the hospital stay, there was a complete physical examination and pain evaluation daily, and liver function tests, serum creatinine level, complete blood count, Child-Pugh score, and electrocardiogram were performed before patient discharge. The 1-month scheduled consultation consisted of a complete physical examination, liver function tests, serum creatinine level, complete blood count, Child-Pugh score, and electrocardiogram. A severe toxicity was defined as a grade 4 or $5 \mathrm{AE}$ according to NCI-CTCAE, any other AE that caused a prolongation of hospitalization of $>7$ days, or any hospitalization within the month after TACE possibly, probably, or definitely attributed to TACE. Secondary end points were tumor response according to modified response evaluation criteria in solid tumors (mRECIST) at MRI 1 month after completion of the first cycle of TACE, time to treatment failure, and overall survival.

\section{Statistical Analysis}

Categorical variables were described by percentages. Continuous variables were expressed as means and standard deviations. Because several patients had repeated TACE sessions, biological data from all sessions were not pooled. The serum levels of liver enzymes and complete blood count were compared before and after the first TACE session by the Wilcoxon signed-rank test. TTTF was defined as the time from the first TACE session to TACE discontinuation for any reason, including disease progression, treatment toxicity, patient preference, or death. Overall survival was defined as the time from the first 
A

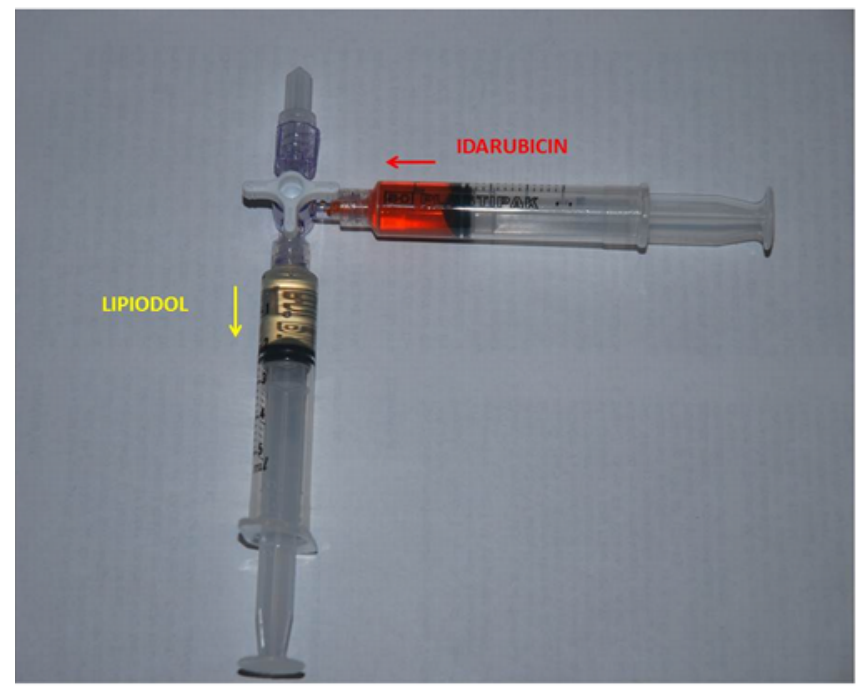

C

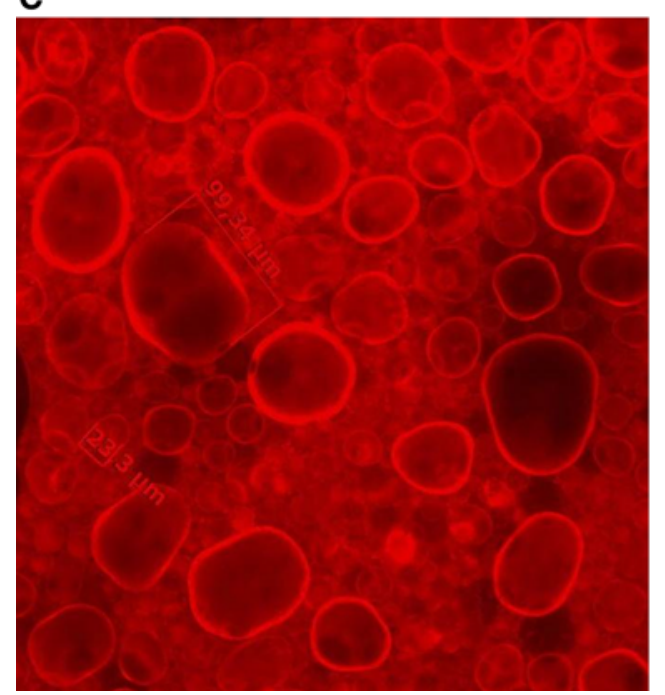

Fig. 1 The emulsion was prepared by mixing an equal volume of iodized oil and $1 \mathrm{mg} / \mathrm{mL}$ idarubicin through a 3-way tap from one $5 \mathrm{~mL}$ syringe to another (10 passages) (A). The physical stability of the idarubicin-ethiodized oil emulsion was examined at $37{ }^{\circ} \mathrm{C}$. Thirty minutes after the preparation (H0.5), the phase separation (arrow) for

TACE session to death (all causes). Survival curves were estimated by the Kaplan-Meier method. All analyses were performed by Stata software, version 10.0 (StataCorp, College Station, TX, USA). A $p$-value below 0.05 was considered significant.

\section{Results}

Baseline Characteristics

The baseline characteristics of the 21 patients are listed in Table 1. Mean age was $68.6 \pm 8.6$ years. All patients were

\section{B}
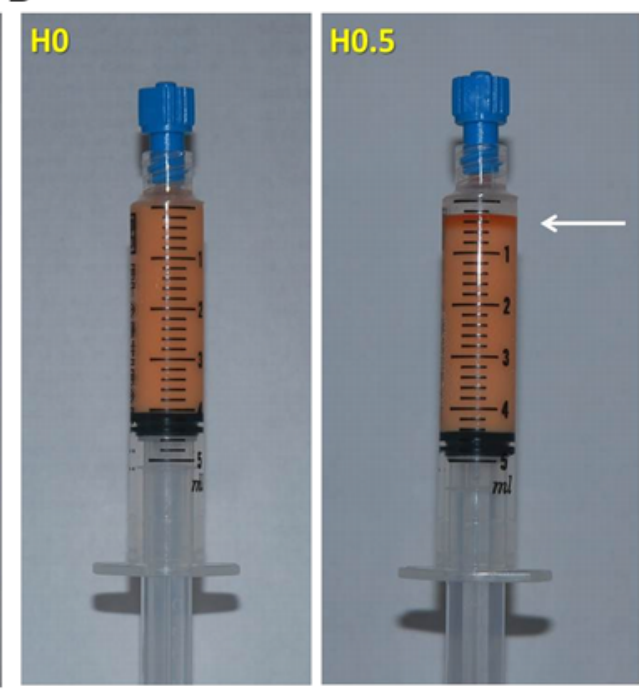

the idarubicin-ethiodized oil emulsion was limited $(5 \%$ aqueous solution and $95 \%$ persisting emulsion) compared with emulsion at H0 (B). Fluorescent micrograph showing the presence of idarubicin (red) on the inner surface of droplets $(\mathbf{C})$. Droplet diameter was $20-100 \mu \mathrm{m}$

cirrhotic; the cause for cirrhosis was alcohol abuse in seven patients $(33.3 \%)$, virus in six patients $(28.6 \%)$, nonalcoholic steatohepatitis (NASH) in three patients (14.3\%), $\mathrm{NASH} /$ virus or alcohol in three patients $(14.3 \%)$, and hemochromatosis in two patients $(9.5 \%)$. All patients had a performance status of $0 / 1$, and the majority had ChildPugh A class liver function (85.7 \%). Most patients ( $81 \%$; 17 of 21) had a multifocal HCC. The mean largest nodule size was $51.3 \pm 23 \mathrm{~mm}$. The mean baseline hemoglobin level, platelet count, and leukocyte count were $14 \pm$ $1.7 \mathrm{~g} / \mathrm{dL}, 157.7 \pm 79.1 \times 10^{3} / \mu \mathrm{L}$, and $6,340 \pm 2,570 / \mathrm{mm}^{3}$, respectively. The mean baseline serum level of liver enzymes was $15.63 \pm 10.8 \mu \mathrm{mol} / \mathrm{L}$ for total bilirubin, 
Table 1 Baseline characteristics of patients and tumors

\begin{tabular}{|c|c|}
\hline Characteristic & Value \\
\hline \multicolumn{2}{|l|}{ Sex } \\
\hline Male & $19(90.5 \%)$ \\
\hline Female & $2(9.5 \%)$ \\
\hline Age (year) & $68.6 \pm 8.6$ \\
\hline \multicolumn{2}{|l|}{ Performance status } \\
\hline $0-1$ & $21(100 \%)$ \\
\hline$\geq 2$ & 0 \\
\hline \multicolumn{2}{|l|}{ Cause of cirrhosis } \\
\hline Virus & $6(28.6 \%)$ \\
\hline Alcohol & $7(33.3 \%)$ \\
\hline NASH & $3(14.3 \%)$ \\
\hline NASH (virus or alcohol) & $3(14.3 \%)$ \\
\hline Hemochromatosis & $2(9.5 \%)$ \\
\hline \multicolumn{2}{|l|}{ Child-Pugh score } \\
\hline A & $18(85.7 \%)$ \\
\hline $\mathrm{B}$ & $3(14.3 \%)$ \\
\hline $\mathrm{C}$ & $0(0 \%)$ \\
\hline \multicolumn{2}{|l|}{ Tumor burden } \\
\hline Unilobar & $4(19 \%)$ \\
\hline Bilobar & $17(81 \%)$ \\
\hline \multicolumn{2}{|l|}{ No. of nodules } \\
\hline $1-3$ & $9(42.9 \%)$ \\
\hline$>3$ & $12(57.1 \%)$ \\
\hline \multicolumn{2}{|l|}{ Tumor size } \\
\hline Size of largest nodule $(\mathrm{mm})$ & $51.3 \pm 23$ \\
\hline Mean total $(\mathrm{mm})$ & $97.6 \pm 62.9$ \\
\hline \multicolumn{2}{|l|}{$\alpha$-Fetoprotein } \\
\hline$<400 \mathrm{ng} / \mathrm{mL}$ & $9(42.9 \%)$ \\
\hline$\geq 400 \mathrm{ng} / \mathrm{mL}$ & $12(57.1 \%)$ \\
\hline \multicolumn{2}{|l|}{ Baseline liver enzymes } \\
\hline AST (U/L) & $58.2 \pm 31.1$ \\
\hline ALT (U/L) & $57.0 \pm 31.4$ \\
\hline $\operatorname{ALP}(\mathrm{U} / \mathrm{L})$ & $128.2 \pm 60.6$ \\
\hline Total bilirubin $(\mu \mathrm{mol} / \mathrm{L})$ & $15.63 \pm 10.8$ \\
\hline Prothrombin $(\%)$ & $76.5 \pm 21.5$ \\
\hline Albumin $(\mathrm{g} / \mathrm{L})$ & $34.4 \pm 4$ \\
\hline GGT & $275.2 \pm 237.6$ \\
\hline \multicolumn{2}{|l|}{ TACE sessions } \\
\hline No. of sessions & 55 \\
\hline Average per patient & $2.62 \pm 1.2$ \\
\hline
\end{tabular}

Data are presented as mean \pm SD or $n(\%)$

$N A S H$ nonalcoholic steatohepatitis, AST aspartate aminotransferase, $A L T$ alanine aminotransferase, $A L P$ alkaline phosphatase, $G G T$ $\gamma$-glutamyltransferase, TACE transarterial chemoembolization

$57.0 \pm 31.4 \mathrm{IU} / \mathrm{L}$ for $\mathrm{ALT}, 58.2 \pm 31.1 \mathrm{IU} / \mathrm{L}$ for $\mathrm{AST}$, $275.2 \pm 237.6 \mathrm{IU} / \mathrm{L}$ for GGT, $128.2 \pm 60.6 \mathrm{IU} / \mathrm{L}$ for ALP, and $76.5 \pm 21.5 \%$ for PT.
TACE Procedure

TACE was performed in all cases without technical failure. A total of 55 sessions were performed, representing 22 TACE cycles. Four patients underwent one session for unilateral disease, 14 underwent two or three sessions, and three underwent more than three sessions for bilateral disease. In all sessions, $100 \%$ of the lipiodol-idarubicin emulsion could be injected. Embolization was conducted with gelfoam in $83.4 \%$ (46 of 55) and $250 \mu \mathrm{m}$ calibrated microspheres in $16.4 \%$ (9 of 55) of sessions.

\section{TACE-Related Toxicity}

Median hospital duration was 4 (range 3-14) days. Postembolization syndrome was observed after $30.9 \%$ (17 of 55) sessions.

No patient died from a TACE-related complication. No hematological grade 3-5 AE was observed. At least one grade 3 or higher AE occurred in $19 \%$ (4 of 21) of patients: one patient experienced a severe TACE-related toxicity (grade 4 with prolonged hospitalization) resulting from severe hypoxia requiring intubation after the second TACE session. Treatment was changed to sorafenib in this patient. Corresponding grade 3 AEs were as follows: one renal failure associated with global cardiac decompensation, one lobar pneumonia requiring antibiotic therapy (this patient was rehospitalized within the month after the TACE session), and hepatic pain necessitating morphine administration during 2 days.

On follow-up imaging, only two patients exhibited very limited liver/biliary toxicity (i.e., segmental bile duct dilatation); no portal vein narrowing or thrombosis or biloma/ liver infarct was observed. At 1 month after the first TACE session, mean hemoglobin level, platelet count, and leukocyte count were $13.6 \pm 1.6 \mathrm{~g} / \mathrm{dL}, 151.8 \pm 70.6 \times 10^{3} / \mu \mathrm{L}$, and $7,700 \pm 2,410 / \mathrm{mm}^{3}$, respectively. At 1 month after the first TACE session, the serum level of liver enzymes was $19.9 \pm 14.2 \mu \mathrm{mol} / \mathrm{L}$ for total bilirubin, $71.2 \pm 70.1 \mathrm{IU} / \mathrm{L}$ for ALT, $58.2 \pm 31.1 \mathrm{IU} / \mathrm{L}$ for AST, $211.5 \pm 151.2 \mathrm{IU} / \mathrm{L}$ for GGT, $180.1 \pm 135.1 \mathrm{IU} / \mathrm{L}$ for ALP, and $78.8 \pm 19.2 \%$ for prothrombin time. These levels (complete blood count and liver enzymes) did not significantly differ from those recorded before TACE, except for GGT (significantly lower after TACE, $p=0.037$ ) and ALP (significantly higher after TACE, $p=0.042$ ).

Response, TTTF, and Overall Survival

One month after the end of the first cycle of TACE, no progression was encountered, four patients (24\%) exhibited stable disease, 12 (57\%) exhibited a partial response, and five (19\%) exhibited complete response (Fig. 2). 

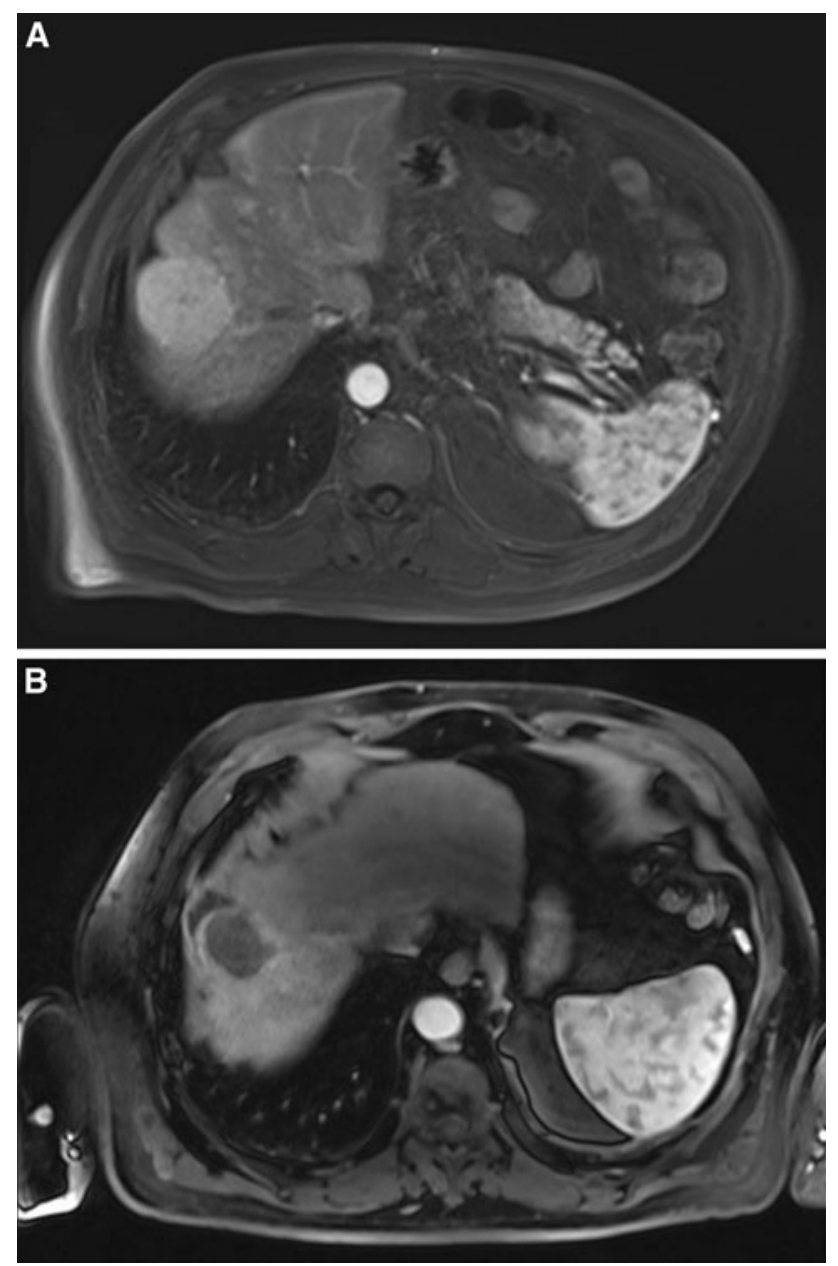

Fig. 2 Five-centimeter unifocal HCC in a 60-year-old patient before (A) and after (B) lipiodol TACE with idarubicin

Median follow-up lasted 8.4 (range 3.2-19) months. Treatment failure occurred before data cutoff in nine patients. Median TTTF was 16.7 months (Kaplan-Meier analysis). At 6 months, $94.7 \%$ (95\% confidence interval [CI] 68.1-99.2) of patients did not reach treatment failure, whereas treatment failure was not reached in $50.6 \%$ (95\% CI 21.6-73.9) of patients at 1 year (Fig. 3). Six patients died before the data cutoff (three of HCC progression, two of fatal variceal bleeding, and one of cirrhosis decompensation). Overall survival was $83.5 \%$ (95\% CI 57-94.4) at 1 year.

\section{Discussion}

To our knowledge, no previous study has been published that used idarubicin injected intra-arterially. Therefore, safety data are crucial. Very few articles have reported reliable toxicity data in conventional TACE. Given its prospective collection of AEs in the two arms (drug-eluting

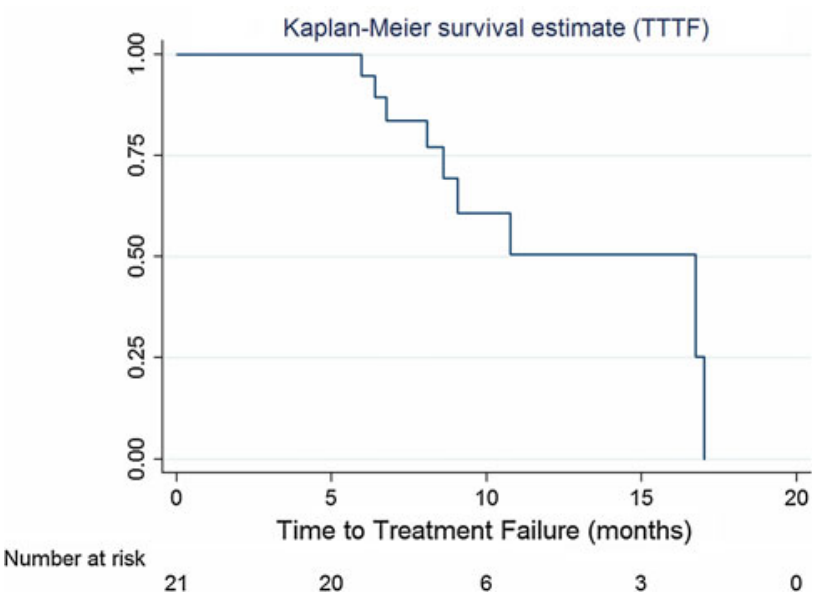

Fig. 3 Time to treatment failure

beads and lipiodol TACE), the phase II randomized PRECISION V trial, which is the largest published study on TACE to date, may serve as a standard for comparison of AEs. In our study, the $19 \%$ occurrence of grade 3 or higher AEs compares favorably to the $29.6 \%$ incidence of serious AEs (defined as events resulting in death; immediately life-threatening; resulting in permanent/significant disability/incapacity; requiring or extending inpatient hospitalization; congenital anomaly/birth defects) reported in the PRECISION V trial [18] with doxorubicin lipiodol TACE. In another article reporting on the same trial [19], the overall incidence of the postembolization syndrome was higher than that reported here ( $72 \%$ vs. $30.9 \%)$. In our study, serum levels of AST, ALT, and bilirubin returned to baseline levels after 1 month, as reported in the PRECISION V study [19]. Unfortunately, no data were provided in this trial regarding serum levels of GGT and ALP. Interestingly, in our series, we did not observe portal vein narrowing, portal veinous thrombosis, or biloma/liver infarct after TACE, whereas such liver/biliary toxicities have been recently reported [17]. Therefore, the toxicity profile of idarubicin used for lipiodol TACE does not seem to be different from that observed with doxorubicin. Although the number of patients is small, the rate of patients with an objective response $(76 \%)$ also compares favorably to those reported with doxorubicin either in the PRECISION V study $(43.5 \%$ in conventional-TACE [CTACE]) [18] or in a large retrospective study on cTACE with doxorubicin, cisplatin, and mitomycin C (64 \%) [20]. However, it should be kept in mind that tumor response has not been validated as a surrogate for overall survival, and that only phase III studies could demonstrate a superiority of idarubicin over doxorubicin for TACE of HCC.

A systematic review demonstrated that the most widely used drugs for TACE were doxorubicin (36\%), cisplatin (31\%), and epirubicin (12\%) [5]. However, until recently, there was no rationale to use one drug over another. Indeed, 
systemic chemotherapy is considered ineffective in HCC $[2,12]$, and thus it cannot help to select the best anticancer agent to use for TACE. Moreover, only two randomized, controlled trials were designed to compare drugs (doxorubicin vs. epirubicin), but they failed to demonstrate better survival $[21,22]$.

We recently compared the cytotoxicity of anticancer agents on human HCC cell lines in order to select the best candidate for TACE [11]. Eleven chemotherapeutic agents were tested, including the most frequently used for TACE. Among them, idarubicin (an anthracycline) was by far the most cytotoxic. The superiority of idarubicin (especially over doxorubicin) was observed most notably in the SNU-449 cell line, known for its resistance to various chemotherapeutic agents [14]. The greater cytotoxicity of idarubicin can be explained by two different mechanisms: first, idarubicin has a higher hepatic penetration compared to other anthracyclines [23]. This may be related to its high lipophilicity, enabling easier intracellular penetration through the cell membrane composed of a double layer of lipids. Second, idarubicin has the ability to overcome the MDR system [24]. The MDR mechanism consists in pumping drugs out of cells and is classically observed in HCC $[13,25,26]$. Both the higher lipophilicity and the ability to overcome MDR could account for a greater accumulation of idarubicin in HCC cells, and therefore a greater efficacy. Interestingly, idarubicin used orally $(5 \mathrm{mg}$ per day for 21-day periods) to treat HCC has been demonstrated to be safe and active [27], but it has not yet been used intra-arterially so far. Yet idarubicin has also an attractive hepatic extraction ratio because the intrahepatic concentration of idarubicin is 1.35 times greater than that of doxorubicin, as demonstrated in an animal model of sarcoma [23].

Idarubicin is a key drug used in hematology to treat acute leukemias [28]; it has a known toxicity profile, mainly hematological and cardiac. Interestingly, in our study, we did not observe any grade 3/4 cardiac or hematological AE. This is not surprising, given the dose used in this series $(10 \mathrm{mg})$. Cardiac toxicities of idarubicin and doxorubicin occur when total cumulative dose are 93 and $550 \mathrm{mg} / \mathrm{m}^{2}$, respectively. For example, with a mean $1.8 \mathrm{~m}^{2}$ patient, it can theoretically be administered 18 sessions of TACE using $10 \mathrm{mg}$ of idarubicin before reaching cumulative cardiac toxicity. Our $10-\mathrm{mg}$ dose is far from the doses of idarubicin used in hematology (commonly $12 \mathrm{mg} /$ $\mathrm{m}^{2}$ each day for three consecutive days, or $8 \mathrm{mg} / \mathrm{m}^{2}$ each day for five consecutive days) which are frequently marrow suppressive. But if we choose to administer only $10 \mathrm{mg}$ idarubicin for lipiodol TACE on the basis of these safety data, we are not sure whether this dose is optimal. In oncology, only phase I studies with escalation of dose allow us to determine the maximum tolerated dose and thus the optimal dose for treatment. However, to our knowledge, this scientific approach has never been used for TACE treatment because no phase I TACE study has been published so far. This lack of phase I TACE studies certainly accounts for the wide variations in dose used for TACE in the literature [5], as is the case, for example, with doxorubicin, the doses of which range 50-150 mg per session.

Although a recent single-center retrospective study performed in Asia demonstrated a benefit in response and survival for drug-eluting beads and TACE over cTACE [29], there is still no level 1 evidence of the superiority of drugeluting beads. Here, we report on conventional TACE using a lipiodol-idarubicin emulsion. We took advantage of the higher lipophilicity of idarubicin, resulting in a great accumulation of the drug in the oily phase, thereby permitting lipiodol to act as a slow-releasing vector. Favoulet et al. [30] demonstrated that lipiodol-doxorubicin emulsion was completely separated into two phases (oil and aqueous) after only $20 \mathrm{~min}$. This might explain the pharmacokinetic data demonstrating that the intra-arterial administration of doxorubicin emulsified with lipiodol has little effect compared with intravenous administration of doxorubicin alone [31]. On the contrary, the phase separation for lipiodolidarubicin emulsion was very limited (5\% aqueous solution and $95 \%$ persisting emulsion); fluorescent electronic microscopy revealed that idarubicin was inside and at the periphery of lipid droplets as a result of drug-lipid ionic interactions. This enhanced emulsion stability is probably an advantage in increasing the contact time of the drug with cancer cells, as previously reported [30].

This study has several limitations: its retrospective design, a small number of patients, and a relatively short follow-up. However, this is a pilot study, not a phase I or II trial. As previously mentioned, we used an empiric dose based on intravenous safety data of idarubicin. This dose might not be the optimal dose. Interestingly, idarubicin can also be loaded in drug-eluting beads, thanks to ionic properties similar to doxorubicin. We are conducting a phase I study to determine the maximum tolerated dose of idarubicin-loaded beads, which will permit us to calculate the optimal dose for idarubicin.

In conclusion, idarubicin seems safe and effective in lipiodol TACE of HCC. This warrants further study to determine the potential of this drug to replace doxorubicin for TACE. We have thus designed a phase II/III study to compare doxorubicin and idarubicin TACE. However, in addition to its promising anticancer efficacy, idarubicin could also be an attractive option to prevent future shortages of doxorubicin.

Acknowledgments We thank Philip Bastable for English-language revision and Olivier Madec for help with data collection. 
Conflict of interest The authors declare that they have no conflict of interest.

\section{References}

1. Guiu B, Minello A, Cottet V et al (2010) A 30-year, populationbased study shows improved management and prognosis of hepatocellular carcinoma. Clin Gastroenterol Hepatol 8:986-991

2. Bruix J, Llovet JM (2009) Major achievements in hepatocellular carcinoma. Lancet 373(9664):614-616

3. EASL-EORTC (2012) EASL-EORTC clinical practice guidelines: management of hepatocellular carcinoma. J Hepatol 56: 908-943

4. Llovet JM, Bruix J (2003) Systematic review of randomized trials for unresectable hepatocellular carcinoma: chemoembolization improves survival. Hepatology 37:429-442

5. Marelli L, Stigliano R, Triantos C et al (2007) Transarterial therapy for hepatocellular carcinoma: which technique is more effective? a systematic review of cohort and randomized studies. Cardiovasc Intervent Radiol 30:6-25

6. Llovet JM, Real MI, Montana X et al (2002) Arterial embolisation or chemoembolisation versus symptomatic treatment in patients with unresectable hepatocellular carcinoma: a randomised controlled trial. Lancet 359(9319):1734-1739

7. Lee KH, Liapi EA, Cornell C et al (2010) Doxorubicin-loaded QuadraSphere microspheres: plasma pharmacokinetics and intratumoral drug concentration in an animal model of liver cancer. Cardiovasc Intervent Radiol 33:576-582

8. Namur J, Wassef M, Millot JM et al (2010) Drug-eluting beads for liver embolization: concentration of doxorubicin in tissue and in beads in a pig model. J Vasc Interv Radiol 21:259-267

9. Malagari K, Pomoni M, Kelekis A et al (2010) Prospective randomized comparison of chemoembolization with doxorubicineluting beads and bland embolization with BeadBlock for hepatocellular carcinoma. Cardiovasc Intervent Radiol 33:541-551

10. Nicolini A, Martinetti L, Crespi S et al (2010) Transarterial chemoembolization with epirubicin-eluting beads versus transarterial embolization before liver transplantation for hepatocellular carcinoma. J Vasc Interv Radiol 21:327-332

11. Boulin M, Guiu S, Chauffert B et al (2011) Screening of anticancer drugs for chemoembolization of hepatocellular carcinoma. Anticancer Drugs 22:741-748

12. Burroughs A, Hochhauser D, Meyer T (2004) Systemic treatment and liver transplantation for hepatocellular carcinoma: two ends of the therapeutic spectrum. Lancet Oncol 5:409-418

13. Minemura M, Tanimura H, Tabor E (1999) Overexpression of multidrug resistance genes MDR1 and cMOAT in human hepatocellular carcinoma and hepatoblastoma cell lines. Int $\mathrm{J}$ Oncol 15:559-563

14. Park JG, Lee SK, Hong IG et al (1994) MDR1 gene expression: its effect on drug resistance to doxorubicin in human hepatocellular carcinoma cell lines. J Natl Cancer Inst 86:700-705

15. Goebel M (1993) Oral idarubicin-an anthracycline derivative with unique properties. Ann Hematol 66:33-43

16. Bruix J, Sherman M (2005) Management of hepatocellular carcinoma. Hepatology 42:1208-1236

17. Guiu B, Deschamps F, Aho S et al (2012) Liver/biliary injuries following chemoembolisation of endocrine tumours and hepatocellular carcinoma: lipiodol vs drug-eluting beads. J Hepatol 56: 609-617
18. Lammer J, Malagari K, Vogl T et al (2010) Prospective randomized study of doxorubicin-eluting-bead embolization in the treatment of hepatocellular carcinoma: results of the PRECISION V study. Cardiovasc Intervent Radiol 33:41-52

19. Vogl TJ, Lammer J, Lencioni R et al (2011) Liver, gastrointestinal, and cardiac toxicity in intermediate hepatocellular carcinoma treated with PRECISION TACE with drug-eluting beads: results from the PRECISION $\mathrm{V}$ randomized trial. AJR Am J Roentgenol 197:W562-W570

20. Lewandowski RJ, Mulcahy MF, Kulik LM et al (2010) Chemoembolization for hepatocellular carcinoma: comprehensive imaging and survival analysis in a 172-patient cohort. Radiology 255:955-965

21. Kawai S, Tani M, Okamura J et al (1997) Prospective and randomized trial of lipiodol-transcatheter arterial chemoembolization for treatment of hepatocellular carcinoma: a comparison of epirubicin and doxorubicin (second cooperative study). The Cooperative Study Group for Liver Cancer Treatment of Japan. Semin Oncol 24(2 suppl 6):S6-38-S36-45

22. Watanabe S, Nishioka M, Ohta Y et al (1994) Prospective and randomized controlled study of chemoembolization therapy in patients with advanced hepatocellular carcinoma. Cooperative Study Group for Liver Cancer Treatment in Shikoku area. Cancer Chemother Pharmacol 33(suppl):S93-S96

23. Broggini M, Italia C, Colombo T et al (1984) Activity and distribution of IV and oral 4-demethoxydaunorubicin in murine experimental tumors. Cancer Treat Rep 68:739-747

24. Roovers DJ, van Vliet M, Bloem AC, Lokhorst HM (1999) Idarubicin overcomes P-glycoprotein-related multidrug resistance: comparison with doxorubicin and daunorubicin in human multiple myeloma cell lines. Leuk Res 23:539-548

25. Berman E, McBride M (1992) Comparative cellular pharmacology of daunorubicin and idarubicin in human multidrug-resistant leukemia cells. Blood 79:3267-3273

26. Vander Borght S, Komuta M, Libbrecht L et al (2008) Expression of multidrug resistance-associated protein 1 in hepatocellular carcinoma is associated with a more aggressive tumour phenotype and may reflect a progenitor cell origin. Liver Int 28: $1370-1380$

27. Tumolo S, Toffoli G, Bearz A et al (2002) Oral idarubicin (IDA) for the treatment of hepatocellular carcinoma (HCC). Paper presented at 2002 ASCO annual meeting

28. Berman E, Heller G, Santorsa J et al (1991) Results of a randomized trial comparing idarubicin and cytosine arabinoside with daunorubicin and cytosine arabinoside in adult patients with newly diagnosed acute myelogenous leukemia. Blood 77: 1666-1674

29. Song MJ, Chun HJ, Song DS et al (2012) Comparative study between doxorubicin-eluting beads and conventional transarterial chemoembolization for treatment of hepatocellular carcinoma. J Hepatol 57:1244-1250

30. Favoulet P, Cercueil JP, Faure P et al (2001) Increased cytotoxicity and stability of lipiodol-pirarubicin emulsion compared to classical doxorubicin-lipiodol: potential advantage for chemoembolization of unresectable hepatocellular carcinoma. Anticancer Drugs 12:801-806

31. Johnson PJ, Kalayci C, Dobbs N et al (1991) Pharmacokinetics and toxicity of intraarterial adriamycin for hepatocellular carcinoma: effect of coadministration of lipiodol. J Hepatol 13: $120-127$ 